\title{
Distributed Sensor Scheduling Scheme Using Euclidean Distance for Large-scale Internet of Things Local Networks
}

\author{
Eui-Jik Kim, ${ }^{1}$ Jaehoon Park, ${ }^{2}$ \\ Yongseok Lim, ${ }^{3 *}$ and Jung-Hyok Kwon ${ }^{1 * *}$ \\ ${ }^{1}$ Department of Convergence Software, Hallym University, \\ 1 Hallymdaehak-gil, Chuncheon-si, Gangwon-do 24252, South Korea \\ ${ }^{2}$ Department of Electronic Engineering, Hallym University, \\ 1 Hallymdaehak-gil, Chuncheon-si, Gangwon-do 24252, South Korea \\ ${ }^{3}$ Smart Network Research Center, Korea Electronics Technology Institute, \\ 11 World Cup buk-ro 54-gil, Mapo-gu, Seoul 03924, South Korea
}

(Received March 30, 2017; accepted March 5, 2018)

Keywords: distributed sensor scheduling, Euclidean distance, Internet of Things local network, redundancy, variables

In this paper, we present a distributed sensor scheduling scheme based on Euclidean distance (DSS-ED) for Internet of Things (IoT) local networks, with the aim of extending the network lifetime while maximizing the utilization of the limited network capacity of the IoT local network to support various IoT applications. For this purpose, the DSS-ED adapts the state of individual sensor devices by comprehensively considering the characteristics of various variables. In the DSS-ED, each sensor device first calculates the Euclidian distance between the measured variables and their ideal values, then notifies its neighbors of the calculated results. Afterward, the sensor device adaptively determines its own state by comparing its Euclidian distance with those of its neighbors. An experimental simulation is conducted to evaluate the performance of the DSS-ED. The results show that the DSS-ED obtains better performance than the lightweight redundancy-aware topology control protocol (LRTCP) in terms of throughput and energy consumption.

\section{Introduction}

Recently, the Internet of Things (IoT) has received considerable attention as a core technology for large-scale monitoring applications such as smart cities, smart factories, and smart farms. ${ }^{(1,2)}$ In IoT applications, a number of resource-constrained sensors constitute the IoT local network, through which the collected data are delivered to a sink or gateway via a lossy wireless link. Thus, the IoT local network is highly similar to the wireless sensor network (WSN) in its structure.

Energy efficiency is a traditionally crucial performance factor for the IoT local network, but it is essential to maximize the utilization of the limited network capacity of the IoT local

\footnotetext{
*Corresponding author: e-mail: busytom@keti.re.kr

** Corresponding author: e-mail: jhkwon@hallym.ac.kr

https://dx.doi.org/10.18494/SAM.2018.1898
} 
network to support various IoT applications. The sensor scheduling mechanism considers the redundancy of sensor devices deployed in the sensing field. It controls their states or actions, thereby extending the network lifetime while maintaining the wireless connectivity for delivering the sensing data. ${ }^{(3-5)}$

To date, many studies have been conducted on sensor scheduling, taking into account the redundancy of sensor devices. Cerpa and Estrin proposed the adaptive self-configuring sensor network topologies (ASCENT) to prolong the network lifetime while maintaining connectivity among sensor devices. ${ }^{(6)}$ For this purpose, ASCENT supports state transition for four states of the sensor devices (i.e., Test, Active, Passive, and Sleep), considering the average packet loss rate and the number of neighbors. However, ASCENT cannot address the load-balancing problem because the sensor device retains its state permanently once it enters the active state. Xie et al. proposed a residual energy-aware scheme (READC) for duty cycle adaptation. This system adapts the sleep and wake states of sensor devices by adjusting the duty cycle of sensor devices, considering their residual energy levels. ${ }^{(7)}$ However, the READC focuses only on the residual energy of sensor devices, thus it may increase the number of lost packets. Zebbane et al. proposed the lightweight redundancy-aware topology control protocol (LRTCP) to improve network connectivity and extend network lifetime. ${ }^{(8)}$ The LRTCP adaptively adjusts the states of sensor devices depending on the degree of redundancy, which is determined by the number of neighbors and the residual energy level of the sensor device. However, the LRTCP does not consider the link quality between the sensor devices, thus degrading the network performance.

In this paper, we propose a distributed sensor scheduling scheme based on Euclidean distance (DSS-ED) for IoT local networks, aiming to extend network lifetime while maximizing the utilization of the limited network capacity of the IoT local network to support various IoT applications. To this end, the DSS-ED adapts the state of individual sensor devices by comprehensively considering the characteristics of various variables. In the DSS-ED, each sensor device first calculates the Euclidian distance between the measured variables and their ideal values, then notifies its neighbors of the calculated result. Afterward, the sensor device adaptively determines its own state by comparing its Euclidian distance and those of its neighbors. To evaluate the performance of the DSS-ED, an experimental simulation is conducted under various scenarios. The results show that the DSS-ED obtains $11.1 \%$ higher throughput and $1.2 \%$ lower energy consumption than those of the LRTCP.

The rest of this paper is organized as follows: the detailed design of the DSS-ED is described in Sect. 2. Section 3 presents the simulation results and performance analysis. Finally, we conclude this paper in Sect. 4.

\section{Design of DSS-ED}

The DSS-ED adapts the state of the sensor devices to extend the network lifetime while maximizing the utilization of the limited network capacity of the IoT local network. The sensor devices are used in various IoT applications with different service requirements. To support various applications, the DSS-ED considers a number of variables related to the network capacity, and the composition of the variable set varies depending on the targeted application. 
Each sensor device maintains a set of variables, as given in

$$
\boldsymbol{V}_{(i)}=\left\{V_{(1, i)}, V_{(2, i)}, \cdots, V_{\left(k_{i}, i\right)}\right\},
$$

where $i$ is the index of each application, $k_{i}$ is the maximum index of the variable for the $i$ th application, $\boldsymbol{V}_{(i)}$ is a set of variables for the $i$ th application, and $V_{\left(k_{i}, i\right)}$ is the $k_{i}$ th variable for the $i$ th application. The sensor devices periodically measure all variables, and update their variable set. The characteristics of the measured variable differ depending on the variable type, thus the sensor device maintains the ideal values for the variable set. These ideal values are compared with each measured variable to check the state of the sensor device. Each ideal value is set to maximize the utilization of the network capacity by the user. For example, if the maximum energy level of the sensor device is $50 \mathrm{~mW}$, its ideal value is set to $50 \mathrm{~mW}$. The ideal value set for $\boldsymbol{V}_{(i)}$ can be expressed as

$$
\boldsymbol{I}_{(i)}=\left\{I_{(1, i)}, I_{(2, i)}, \cdots, I_{\left(k_{i}, i\right)}\right\},
$$

where $I_{\left(k_{i}, i\right)}$ is the ideal value for $V_{\left(k_{i}, i\right)}$.

Then, the sensor device calculates the Euclidean distance between the measured variables and their ideal values. For this, the sensor device first conducts a normalization to convert both the measured variables and the ideal values to values between 0 and 1 , where the larger value is set to 1 and the smaller value is set to a ratio proportional to the larger value. Table 1 shows an example of the normalization in which the measured variable and ideal value for the residual energy are converted to 0.5 and 1 , respectively. In the case of the number of neighbors, the measured variable and the ideal value are converted to 1 and 0.2 , respectively.

After normalization, the sensor device calculates the Euclidean distance between two converted values, namely, the normalized measured variables and the normalized ideal values, which are calculated with

$$
D_{(l, i)}=\sqrt{\sum_{j=1}^{k_{i}}\left(V_{(j, i)}^{n o r m}-I_{(j, i)}^{\text {norm }}\right)^{2}},
$$

where $D_{(l, i)}$ is the Euclidean distance of the $l$ th sensor device for the $i$ th application, and $V_{(j, i)}^{n o r m}$

Table 1

Example of normalization.

\begin{tabular}{lcccc}
\hline \multirow{2}{*}{ Variable type } & \multicolumn{2}{c}{ Before normalization } & \multicolumn{2}{c}{ After normalization } \\
\cline { 2 - 5 } & Measured variable & Ideal value & Measured variable & Ideal value \\
\hline Residual energy & $50 \mathrm{~W}$ & $100 \mathrm{~W}$ & 0.5 & 1 \\
Number of neighbors & 10 & 2 & 1 & 0.2 \\
Link quality & $10 \mathrm{Mbps}$ & $100 \mathrm{Mbps}$ & 0.1 & 1 \\
\hline
\end{tabular}


and $I_{(j, i)}^{\text {norm }}$ are the normalized values for the $j$ th measured variables and ideal values for the $i$ th application, respectively. Then, the sensor device periodically informs the neighbors of their calculated Euclidean distance. Upon receiving the Euclidean distance from their neighbors, each sensor device maintains its list of Euclidean distances in a table and counts the number of neighbors. The table and the number of neighbors are used to determine the state of each sensor device.

Figure 1 shows the state transition diagram for each sensor device. The sensor device has the following four states: Test, Active, Passive, and Sleep, and sets the timer in each state for the state transition. In the figure, the timers for Test, Active, Passive, and Sleep states are expressed as $T_{t}, T_{a}, T_{p}$, and $T_{s}$, respectively. Test is the initial state of the sensor device, where the sensor device exchanges control messages including the Euclidean distance. If the number of Active neighbors is less than or equal to the neighbor threshold (NT) and the Euclidean distance is the smallest among the neighbors, the sensor node checks the timer $T_{t}$ to change its state to the Active state. Otherwise, the state is changed to the Passive state. The NT is predefined by the user and refers to the maximum number of Active neighbors. In the Active state, the sensor device detects changes in the surrounding environment, and transmits the sensed data to the sink. When the timer $T_{a}$ expires, the sensor device changes its state to the Test state. On the other hand, the sensor device turns on its radio only to listen to the neighbors' control messages while in the Passive state. In this state, the sensor device changes its state to Sleep when the timer $T_{p}$ expires. The sensor device turns off its radio to save energy in the Sleep state. When the timer $T_{S}$ expires in the Sleep state, the sensor device changes its state to the Passive state.

\section{Performance Evaluation}

A simulation is conducted to evaluate the performance of the DSS-ED. To verify the effectiveness of the DSS-ED, the simulation results are compared with those of the LRTCP. In the simulation, the sensor devices are randomly placed in a $100 \times 100 \mathrm{~m}^{2}$ area, each of which transmits $1024 \mathrm{~B}$ of data toward the sink. We consider four variables, namely, the distance from

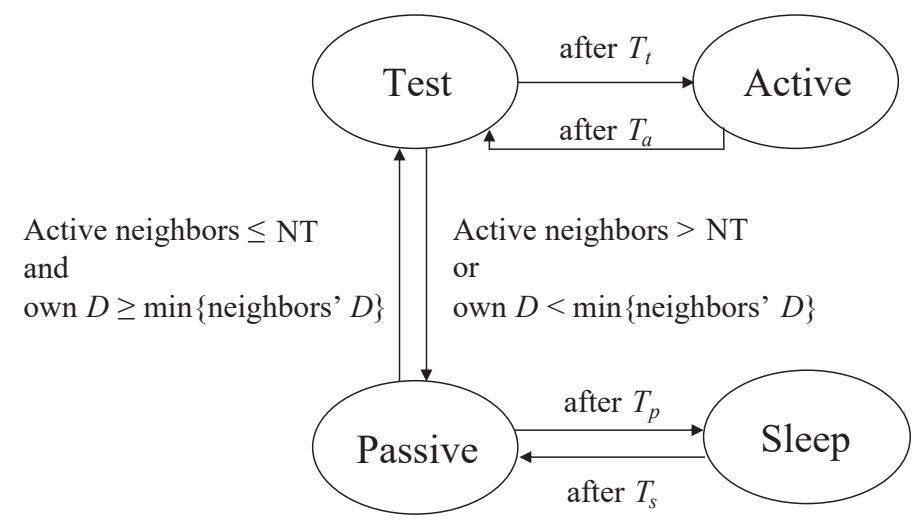

Fig. 1. State transition diagram. 
the sink, the residual energy, the number of neighbors, and the link quality, whose ideal values are assumed to be $0 \mathrm{~m}, 100 \mathrm{~W}, 3$, and $250 \mathrm{kbps}$, respectively. Regarding the variables, the DSSED uses the four different variables, while only two variables (i.e., the residual energy and the number of neighbors) are adopted in the LRTCP. The simulation parameters are listed in Table 2.

Figure 2 shows the variation of the throughput when the number of sensor devices increases. Overall, the DSS-ED obtains higher throughput than the LRTCP. This is because the DSSED additionally considers the link quality between neighbors and the distance from the sink to determine the state of the sensor device, thereby reducing the packet loss rate and the number of hop counts. On average, the DSS-ED obtains $11.1 \%$ higher throughput than the LRTCP. In both cases, if the number of sensor devices is less than 30, the throughput increases sharply as the number of sensor devices increases. However, when the number of sensor devices exceeds 30 , the throughput becomes almost constant. The reason is that the sensor devices change their state to the Sleep state as the number of active neighbors reaches the NT.

Figure 3 shows the variation in energy consumption for each sensor device as the number of sensor devices increases. Both the DSS-ED and LRTCP exhibit similar curves because the number of neighbors and the remaining energy are commonly taken into account as variables in both cases. However, the energy consumption of the DSS-ED is slightly lower than that of the LRTCP. More specifically, the DSS-ED achieves a 1.2\% lower energy consumption than

Table 2

Simulation parameters.

\begin{tabular}{lccc}
\hline Parameter & Value & Parameter & Value \\
\hline MAC/PHY model & IEEE 802.15 .4 & MAC header & $15 \mathrm{~B}$ \\
Data rate & $250 \mathrm{kbps}$ & Payload & $1003 \mathrm{~B}$ \\
Size of the sensing field & $500 \times 500 \mathrm{~m}^{2}$ & Idle energy consumption & $16.4 \mathrm{~mW}$ \\
Number of sensor nodes & 100 & $T x$ energy consumption & $17.9 \mathrm{~mW}$ \\
Transmission range & $20 \mathrm{~m}$ & $R x$ energy consumption & $15.7 \mathrm{~mW}$ \\
PHY header & $6 \mathrm{~B}$ & Sleep energy consumption & $0.2 \mu \mathrm{W}$ \\
\hline
\end{tabular}

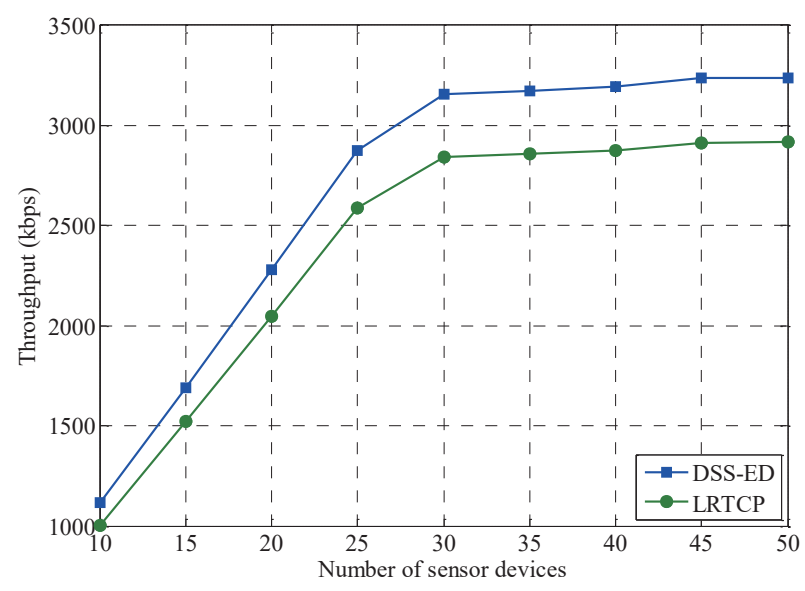

Fig. 2. (Color online) Throughput.

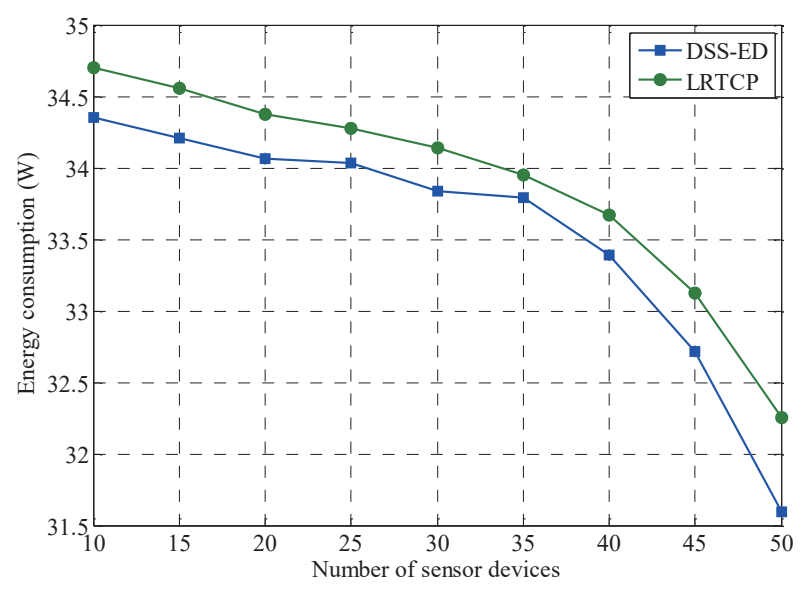

Fig. 3. (Color online) Energy consumption. 
does the LRTCP. The reason is that the DSS-ED increases the idle time of the sensor device by keeping the sensor devices with better link quality in the Active state, unlike the LRTCP.

\section{Conclusions}

In this paper, we proposed a DSS-ED for IoT local networks. To support various IoT applications, the DSS-ED adapts the state of individual sensor devices by comprehensively considering the characteristics of various variables. The DSS-ED is aimed at extending network lifetime while maximizing the utilization of the limited network capacity of the IoT local network. Therefore, in the DSS-ED, each sensor device calculates the Euclidian distance between the measured variables and their ideal values. Then, the sensor device adaptively determines its own state by comparing its Euclidian distance with those of its neighbors. To evaluate the performance of the DSS-ED, we conducted simulation using the IEEE 802.15.4 network model to compare the performance of the DSS-ED with that of the LRTCP. The results showed that the DSS-ED obtains $11.1 \%$ higher throughput than the LRTCP since it additionally considers the link quality between neighbors and the distance from the sink. In addition, the DSS-ED increases the idle time of the sensor device by keeping the sensor devices with better link quality in the active state, unlike the LRTCP, thus achieving $1.2 \%$ lower energy consumption than the LRTCP.

\section{Acknowledgments}

This work was supported by the Hallym Leading Research Group Support Program of 2017 (HRF-LGR-2017-0003).

\section{References}

1 W. Ejaz, M. Naeem, A. Shahid, A. Anpalagan, and M. Jo: IEEE Commun. Mag. 55 (2017) 84.

2 Y.-B. Lin, Y.-W. Lin, C.-Y. Chih, T.-Y. Li, C.-C. Tai, Y.-C. Wang, F. J. Lin, H.-C. Kuo, C.-C. Huang, and S.-C. Hsu: IEEE Internet of Things J. 2 (2015) 551.

3 J. Huang, Q. Duan, C.-C. Xing, and H. Wang: IEEE Wireless Commun. 24 (2017) 67.

4 H. P. Gupta, S. V. Rao, and T. Venkatesh: IEEE Trans. Veh. Technol. 65 (2016) 8423.

5 B. Zhang, E. Tong, and J. Hao: J. Network Syst. Manage. 24 (2016) 834.

6 A. Cerpa and D. Estrin: IEEE Trans. Mob. Comput. 3 (2004) 272.

7 R. Xie, A. Liu, and J. Gao: Wireless Pers. Commun. 90 (2016) 1859.

8 B. Zebbane, M. Chenait, and N. Badache: Wireless Networks 23 (2017) 1779. 